\title{
Efecto de una actividad grupal educativa en la calidad de vida de la persona con EPOC
}

\section{Effect of an educational group activity on the quality of life of persons with COPD}

\section{Efeito de uma atividade grupal educativa na qualidade de vida da pessoa com DPOC}

\author{
L.F. Achury-Beltrán ${ }^{\mathrm{a} *}$, P. Garcia-Peñuela ${ }^{\mathrm{b}}$ \\ ORCID: \\ a $0000-0001-6931-396 \mathrm{X}$ \\ b 0000-0002-7637-3722 \\ Facultad de Enfermería, Pontificia Universidad Javeriana, Bogotá, Colombia. \\ Recibido: 17 noviembre 2018 \\ Aceptado: 28 marzo 2019
}

\section{Resumen}

Introducción: La enfermedad pulmonar obstructiva crónica (EPOC), es un importante problema de salud pública que repercute sobre la calidad de vida. Se requieren intervenciones que reduzcan su impacto. Objetivo: Determinar el efecto de una actividad educativa grupal sobre la calidad de vida de personas con EPOC, que asisten a una institución de cuarto nivel durante septiembre-2017 y junio-2018, en Bogotá, Colombia.

Métodos: Diseño cuasi-experimental. La variable independiente fue la actividad educativa grupal y la variable dependiente la calidad de vida, medida con el Cuestionario Respiratorio de Saint George (SGRQ). Los pacientes se aleatorizaron con una tabla generada por computador. El grupo control $(n=30)$ recibió la intervención usual y el grupo experimental $(n=30)$ una actividad educativa grupal diseñada bajo recomendaciones internacionales, impartida por el personal de enfermería. Se excluyeron pacientes con asistencia a actividades grupales durante los últimos dos meses.

Resultados: La media de la calidad de vida pos-intervención fue $41 \%$ y $32 \%$ para el grupo control y experimental, respectivamente, se redujeron dos puntos con respecto a la medición inicial. La dimensión de actividad fue la más comprometida. No se encontraron diferencias estadísticamente significativas en el análisis intragrupal ni intergrupal. 
Discusión: La leve mejoría pos-intervención en las dimensiones de actividad e impacto, así como las variables sociodemográficas son congruentes con otros estudios. Los resultados pueden guardar relación con la cantidad de actividades desarrolladas.

Conclusiones: La actividad grupal no genera mejoría estadísticamente significativa en la calidad de vida de las personas con EPOC. Se identificó una mejoría clínica en las dimensiones de actividad e impacto, así como en la puntuación global.

Palabras clave: Educación; enfermedad pulmonar obstructiva crónica; calidad de vida; enfermería; Colombia.

\section{Abstract}

Introduction: Chronic obstructive pulmonary disease (COPD) is an important public health problem which affects the quality of life. Consequently, interventions aimed at reducing these impacts are necessary.

Objective: To determine the effect of an educational group activity on the quality of life of persons with COPD being attended in a fourth level institution in Bogota, Colombia, in september, 2017 and june, 2018.

Methods:The study design was quasi-experimental. The independent variable was the educational group activity, and the dependent variable was the quality of life measured by the St George's Respiratory Questionnaire (SGRQ). Patients were randomized using a computer generated table. The control group $(n=30)$ received the usual intervention while the experimental group $(n=30)$ had an educational group activity designed under international recommendations and given by the nursing staff. Patients who had had other group activities during the last two months were excluded.

Results: The post intervention quality of life mean was $41 \%$ and $32 \%$ for the control and experimental groups respectively. The most compromised dimension was activity. No statistically significant differences were found in the within-group and between-group analyzes.

Discussion: The slight post intervention improvement in the dimensions of activity and impact is consistent with other similar studies, but results might vary in relation to the activities performed.

Conclusions: This group activity did not induce a statistically significant improvement in the overall quality of life of persons living with COPD, nevertheless, slight improvements were found in the dimensions of activity, impact, and total score.

Keywords: Education; chronic obstructive pulmonary disease; quality of life; nursing; Colombia.

\section{Resumo}

Introdução: A doença pulmonar obstrutiva crónica (DPOC), é um importante problema de saúde pública que tem influência sobre a qualidade de vida. Requerem-se intervenções que reduzam seu impacto. Objetivo: Determinar o efeito de uma atividade educativa grupal sobre a qualidade de vida de pessoas com DPOC, que assistem a uma instituição de quarto nível durante setembro-2017 e junho-2018, em Bogotá, Colômbia.

Métodos: Desenho quase-experimental. A variável independente foi a atividade educativa grupal e a variável dependente, a qualidade de vida, medida com o Questionário Respiratório de Saint George (SGRQ). Os pacientes foram aleatorizados com uma tabela gerada por computador. O grupo controle $(\mathrm{n}=30)$ recebeu a intervenção usual e o grupo experimental $(n=30)$ uma atividade educativa grupal desenhada sob recomendações internacionais, ministrada pelo pessoal de enfermagem. Excluíram-se pacientes com assistência a atividades grupais durante os últimos dois meses.

Resultados: A média da qualidade de vida pós- intervenção foi 41\% e 32\% para o grupo controle e experimental, respetivamente, reduziram-se dois pontos ao respeito da medição inicial. A dimensão de atividade foi a mais comprometida. Não se encontraram diferenças estatisticamente significativas na análise intragrupal nem na intergrupal. 
Discussão: A leve melhora pós-intervenção nas dimensões de atividade e impacto, assim como as variáveis sociodemográficas são congruentes com outros estudos. Os resultados podem guardar relação com a quantidade de atividades desenvolvidas.

Conclusões: A atividade grupal não gera melhora estatisticamente significativa na qualidade de vida das pessoas com DPOC. Encontrou-se uma melhora clínica nas dimensões de atividade e impacto, assim como na pontuação global.

Palavras chave: Educação; doença pulmonar obstrutiva crónica; qualidade de vida; enfermagem; Colômbia.

\section{Introducción}

La enfermedad pulmonar obstructiva crónica (EPOC), se caracteriza por la persistencia de síntomas respiratorios y una limitación al flujo del aire, provocada por anormalidades alveolares o en la vía aérea, que usualmente es causada por la exposición a partículas nocivas o gases, en combinación con una variedad de factores genéticos, de hiperreactividad de la vía aérea y de pobre desarrollo pulmonar durante la infancia ${ }^{1}$.

De acuerdo con las estadísticas reportadas por el grupo Global Burden of Disease, a nivel mundial la EPOC es la causa del $5.36 \%$ del total de muertes y del $2.06 \%$ de años vividos con discapacidad ${ }^{1}$; en Colombia, es la responsable del $6.01 \%$ del total de muertes y del $0.81 \%$ de años vividos con discapacidad ${ }^{2}$, que la ubica como la tercera causa de mortalidad a nivel mundial, en el grupo de las enfermedades crónicas no trasmisibles ${ }^{3}$. Este aspecto desencadena un grave problema de salud pública, no sólo por el impacto que la EPOC genera sobre la morbimortalidad de la población, sino también por la importante carga económica y social que desata, dado que se asocia a un incremento en los gastos derivados de los cuidados informales otorgados por familiares y amigos, así como la perdida de la productividad laboral ${ }^{4}$.

Este panorama se relaciona con las consecuencias derivadas de la enfermedad, puesto que la cronicidad y progresión de la EPOC favorece en los pacientes la aparición de síntomas como disnea, tos, sibilancias, sensación de opresión torácica, en casos severos de la enfermedad, fatiga, pérdida de peso y anorexia, los cuales trascienden, de manera negativa sobre el desarrollo de las actividades de la vida diaria $^{5}$, generan repercusiones a nivel funcional, emocional y social ${ }^{6}$, por lo que reducen significativamente la calidad de vida de la persona ${ }^{7}$, esta es definida como una experiencia individual subjetiva acerca del efecto de la enfermedad y su tratamiento sobre la función de los individuos, por lo que se relaciona con las repercusiones que pueden observarse sobre las dimensiones física, emocional, mental, económica, familiar, social y de bienestar8.

Desde el ámbito emocional, los pacientes experimentan ansiedad, temor frente a la muerte, incertidumbre, desesperanza, resignación y, en varias ocasiones episodios depresivos derivados de la limitación física, las hospitalizaciones recurrentes y la pérdida acelerada de la función pulmonar?. Por otra parte, en el ámbito social, la pérdida gradual de la autonomía trasforma la relación con su entorno y su familia, e incrementa con ello la dependencia ${ }^{10}$, lo que hace que el cuadro de la enfermedad se convierta en una secuencia de eventos que terminan magnificando el impacto de la EPOC, sobre el desempeño de quienes lo padecen y con ello se deteriore la calidad de vida.

Por esta razón, la búsqueda de intervenciones que minimicen las repercusiones de la enfermedad se ha convertido en un aspecto frecuente de investigación en enfermería, con ello, la educación para la salud ha cobrado relevancia como parte del tratamiento de los pacientes con EPOC. De acuerdo a diferentes lineamientos nacionales e internacionales ${ }^{1,11}$, la educación para el paciente que padece de EPOC, debe estar prioritariamente orientada hacia el conocimiento de la enfermedad y su tratamiento, con el objetivo de incrementar la adherencia al régimen terapéutico, disminuir la ansiedad, reducir el impacto psicosocial, facilitar la identificación precoz de las exacerbaciones, incrementar la calidad de vida, reducir las hospitalizaciones y con ello la demanda, así como los costos de los servicios de salud, tal como lo plantean Soler et al. ${ }^{12}$. 
Además, la educación para la salud se fundamenta en una adecuada comunicación en donde el mensaje emitido por el transmisor debe llegar a las tres esferas de la conciencia del receptor (cognoscitiva, afectiva y volitiva), con el fin de obtener el cambio de conducta permanente que se desea. Una modalidad de la educación para la salud consiste en el desarrollo de actividades educativas grupales, las cuales han demostrado efectos benéficos en los pacientes con enfermedades crónicas, puesto que el refuerzo positivo entre los asistentes, facilita el proceso de cambio de conducta y la adopción de estilos de vida saludables, lo cual permite que cada participante lleve su propio proceso y ritmo terapéutico ${ }^{13}$.

Algunos estudios que han implementado ésta estrategia terapéutica obtuvieron efectos positivos, por ejemplo, el estudio de Alves et al. ${ }^{14}$ encontró que la educación grupal incrementa de forma significativa el conocimiento en pacientes con diabetes mellitus. De forma similar, Fernández y Manrique ${ }^{15}$ en su estudio, hallaron un incremento en la capacidad de agencia de autocuidado de pacientes hipertensos, tras la asistencia a educación grupal; de igual manera en el estudio de Rodríguez Martín et al. ${ }^{16}$, se advirtió un cambio positivo en los estilos de vida de personas con hipertensión, aspectos que producen una mejoría en los desenlaces clínicos.

Algunos estudios llevados a cabo en pacientes con EPOC mostraron una reducción de los ingresos hospitalarios y las consultas a urgencias de estos pacientes, así como un incremento en los conocimientos de la enfermedad y la técnica inhalatoria ${ }^{17,18}$; sin embargo, la heterogeneidad de las intervenciones realizadas y reportadas por los recientes metaanálisis ${ }^{19}$, ${ }^{20}$, así como la incapacidad de extrapolar los resultados de los estudios internacionales a nuestra población (esto por las particularidades en las características tanto sociodemográficas como ambientales), además de la insuficiente evidencia en el contexto colombiano, acerca del efecto de una actividad educativa grupal sobre la calidad de vida de la persona con EPOC, direccionaron la siguiente pregunta de investigación: ¿cuál es el efecto de una actividad educativa grupal en la calidad de vida del paciente con EPOC, que asiste a una institución de cuarto nivel de atención durante el periodo comprendido entre septiembre de 2017 y junio de 2018 ?

\section{Metodología}

Diseño. Se utilizó un diseño cuasi-experimental cuya variable independiente fue la actividad educativa y la variable dependiente, la calidad de vida. El grupo experimental participó en una actividad educativa grupal llevada a cabo por enfermería, diseñada a partir de las recomendaciones de la Sociedad Española de Neumología en sus guías de práctica clínica ${ }^{21}$ y contempló temáticas relacionadas con la enfermedad, las técnicas de conservación de energía, los medicamentos, el reconocimiento de las exacerbaciones, el manejo de la ansiedad y el estrés, mientras que el grupo control recibió la intervención usual de la institución de salud, que consistió en la educación individual brindada durante la consulta médica.

Población de estudio y tamaño de muestra. La población estuvo conformada por los individuos con diagnóstico de EPOC por historia clínica y que asistieron a consulta externa, control de medicina interna o neumología, en una institución de cuarto nivel de atención en Bogotá-Colombia, durante el periodo comprendido entre el mes de septiembre de 2017 y junio de 2018.

La variable usada para el cálculo del tamaño de la muestra, fue la mejoría de la calidad de vida de acuerdo con el Cuestionario Respiratorio Saint George (SGRQ), con el cual se pretendió detectar una diferencia de cuatro unidades entre los puntajes de calidad de vida de los dos grupos, dado que éste es el valor que la literatura reporta como clínicamente significativo ${ }^{22}$, por lo tanto, el tamaño de la muestra calculada con un poder del $80 \%$ y un riesgo de error de $0.05 \%$, correspondió a 60 pacientes, 30 para cada uno de los grupos.

La asignación al grupo experimental y al grupo control se realizó utilizando un muestreo probabilístico, con un método aleatorio simple, a través de una tabla de números aleatorios entre 0 y 1 generada por computador. Los pacientes candidatos a ingresar al estudio fueron incluidos a cada uno de los grupos: control (0) y experimental (1), de acuerdo al orden de la lista del computador. 
Criterios de inclusión. Pacientes con EPOC en cualquier estadio de clasificación, que asistían a una institución de cuarto nivel de atención en Bogotá-Colombia, que voluntariamente decidieron participar en el estudio con previa firma del consentimiento informado, que no hubieran presentado exacerbaciones durante el último mes, ni participado en actividades educativas grupales durante un periodo de dos meses previos a la intervención.

Criterios de exclusión. Pacientes que padecieran de algún grado de afección del sistema nervioso que determinara un deterioro motor, pacientes con déficit cognitivo, patologías mentales y en etapa terminal.

Intervención. La actividad educativa se fundamentó, por una parte en las recomendaciones del grupo de trabajo de la GesEPOC ${ }^{21}$ y por otra, en el referente teórico de Imogene King ${ }^{23}$, con el que se pretende la consecución de los objetivos del paciente, a partir de una interacción enfermera-paciente (en donde el paciente expone sus experiencias, percepciones y necesidades, mientras que la enfermera aporta sus conocimientos), proceso en el cual la comunicación es esencial, ya que permite la transmisión de la información y el intercambio.

La actividad educativa se realizó con grupos de entre dos y cinco pacientes, los cuales a partir de una metodología participativa contaron sus experiencias, recibieron información con ayudas audiovisuales y participaron en talleres prácticos sobre las siguientes temáticas:

- La EPOC en nuestra vida: definición de la EPOC, causas, signos, síntomas e impacto de la enfermedad en el desarrollo de actividades laborales, sociales, familiares y las relacionadas con la sexualidad.

- Aprendiendo a respirar y a conservar nuestra energía: definición y explicación de la importancia de la técnica de respiración en la enfermedad y las técnicas de conservación de la energía. Taller: técnicas de respiración y de conservación de energía.

- Nuestros medicamentos: generalidades de los medicamentos utilizados, técnicas de inhalación y taller práctico de uso de inhaladores e inhalocámara.

- Reconociendo oportunamente los factores que afectan nuestra salud: prevención, reconocimiento oportuno y manejo de las exacerbaciones e infecciones respiratorias. Reconocimiento y reducción de los factores ambientales.

- Manejando la ansiedad y el estrés: definición del estrés y la ansiedad, así como la importancia de la incorporación de técnicas de relajación.

La actividad educativa tuvo una duración una hora con 30 minutos, para cada una de las temáticas se diseñó una cartilla educativa, las cuales fueron entregadas al finalizar la sesión, para que el paciente pudiera reforzar la información las veces que lo necesitara.

Instrumento. Para la recolección de la información se utilizó el cuestionario SGRQ ${ }^{24}$, el cual determina la calidad de vida de los pacientes con EPOC durante las últimas cuatro semanas. El instrumento consta de 50 preguntas, 10 de las cuales son de opción múltiple y 40 de falso o verdadero, dividido en tres dimensiones:

1. Síntomas: constituida por 8 preguntas que evalúan toda la sintomatología de la enfermedad pulmonar, así como su frecuencia y severidad.

2. Actividades: estructurada en 16 preguntas que evalúan la limitación en la realización de actividades debida a la aparición de disnea.

3. Impacto: conformada por 26 preguntas que se refieren a situaciones o aspectos relacionados con el funcionamiento social o psicológico, que pueden verse afectados por la enfermedad pulmonar.

El cálculo del puntaje de cada individuo se realiza inicialmente por dimensiones, para finalmente, poder obtener el puntaje total cuyo rango oscila entre 0-100\%. A menor porcentaje, mayor calidad de vida.

El instrumento ha sido traducido en diferentes idiomas y la versión en español tiene un alfa de Cronbach de 0.94 para la totalidad de la escala, 0.72 para la sub-escala de Síntomas, 0.89 para la de Actividad y 0.89 para la de Impacto, por lo tanto demuestra su validez y confiabilidad ${ }^{24}$. De la misma manera, ha 
sido utilizada en diferentes poblaciones incluso la población colombiana, razón por la cual fue seleccionada para el presente estudio.

Procedimiento. Con la previa firma del consentimiento informado, los pacientes seleccionados para el estudio fueron asignados a cada uno de los grupos. Al inicio del estudio tanto al grupo control como al experimental se les aplicó el Cuestionario SGRQ y el formato de variables sociodemográficas que contempló: edad, género, escolaridad, estado civil, estrato socioeconómico, red de apoyo, uso de medicamentos y oxigeno como parte del tratamiento.

Los pacientes del grupo experimental participaron en una actividad educativa grupal y un mes posterior a la primera medición, los pacientes de los dos grupos fueron medidos nuevamente con el Cuestionario SGRQ, para comparar mediante las mediciones pos- intervención de los dos grupos la calidad de vida y el efecto de la intervención.

Procesamiento y análisis de datos. Los datos obtenidos del diligenciamiento de los instrumentos fueron transcritos a la calculadora de Excel, proporcionada por los autores del instrumento, para realizar el cálculo de las dimensiones y la puntuación total.

Se usaron herramientas descriptivas para el análisis de las variables sociodemográficas y la prueba T Student, para la evaluación cuantitativa del efecto de la actividad educativa, esto cuando los resultados se ajustaron al modelo normal posterior a la aplicación de la prueba de Shapiro-Wilk. Para las dimensiones en las cuales no se cumplió el supuesto de normalidad, se aplicaron test no paramétricos equivalentes como la prueba U de Mann-Whitney y el test de Wilcoxon de rango signado.

Consideraciones éticas. Se tuvieron en cuenta la Resolución 008430 de 1993 del entonces Ministerio de Salud de la República de Colombia y las pautas éticas del Consejo de Organizaciones de Ciencias Médicas y la Organización Mundial de la Salud (OMS). Adicionalmente, el estudio fue avalado por el comité de ética e investigación de la institución participante. Se mantuvo la confidencialidad de la información y el anonimato de los participantes, se le identificó a cada uno, con un número consecutivo durante el estudio.

\section{Resultados}

Los resultados del estudio describen por una parte las variables sociodemográficas evaluadas y por otra, el efecto de la intervención a partir de un análisis intergrupal e intragrupal. El análisis intergrupal compara los resultados del grupo control y experimental, mientras que el análisis intragrupal compara los resultados de las mediciones pre y post intervención al interior de cada uno de los grupos.

\section{Análisis de las variables sociodemográficas}

Como se muestra en la Tabla 1, la mayoría de los pacientes en ambos grupos son mujeres, están casados, tienen como máximo nivel educativo el bachillerato, pertenecen a estratos socioeconómicos 1,2 y 3 (los estratos 1 y 2 se consideran bajos, 3 y 4 medios), los cuales corresponden a un nivel medio-bajo y su principal red de apoyo son los hijos. La mayoría de estas personas no han asistido previamente a actividades educativas estructuradas respecto a la enfermedad, usan medicamentos y oxígeno como parte de los tratamientos.

Con respecto a la variable sociodemográfica de edad, la Tabla 2 muestra para el grupo experimental una edad que oscila entre los 54 y 90 años, mientras que en el grupo control se encuentra entre los 61 y 89 años, con una media de 73 y 75 años, respectivamente, lo cual es congruente con la edad de aparición de la enfermedad y la esperanza de vida media en Colombia. 
Tabla 1. Variables sociodemográficas de los participantes del estudio

\begin{tabular}{|c|c|c|c|}
\hline \multicolumn{2}{|c|}{ Variable } & $\begin{array}{c}\text { Grupo experimental } \\
\%\end{array}$ & $\begin{array}{c}\text { Grupo control } \\
\%\end{array}$ \\
\hline \multirow[t]{2}{*}{ Sexo } & Masculino & 43 & 37 \\
\hline & Femenino & 57 & 63 \\
\hline \multirow[t]{5}{*}{ Estado civil } & Casado & 67 & 67 \\
\hline & Divorciado & 3 & 10 \\
\hline & Soltero & 3 & 0 \\
\hline & Unión libre & 7 & 0 \\
\hline & Viudo & 20 & 23 \\
\hline \multirow[t]{5}{*}{ Escolaridad } & Ninguno & 3 & 3 \\
\hline & Primaria & 27 & 44 \\
\hline & Bachillerato & 47 & 50 \\
\hline & Técnico & 17 & 3 \\
\hline & Universitario & 6 & 0 \\
\hline \multirow[t]{4}{*}{ Estrato socioeconómico } & 1 & 3 & 7 \\
\hline & 2 & 40 & 56 \\
\hline & 3 & 47 & 37 \\
\hline & 4 & 10 & 0 \\
\hline \multirow[t]{7}{*}{ Red de apoyo } & Ninguno & 0 & 3 \\
\hline & Exesposo (a) & 3 & 0 \\
\hline & Esposo(a) & 23 & 10 \\
\hline & Hijos & 54 & 77 \\
\hline & Hermanos & 0 & 7 \\
\hline & Esposo(a) e hijos & 17 & 3 \\
\hline & Otro miembro de la familia & 3 & 0 \\
\hline \multirow{2}{*}{$\begin{array}{l}\text { Asistencia previa a } \\
\text { actividades educativas }\end{array}$} & Sí & 3 & 0 \\
\hline & No & 97 & 100 \\
\hline \multirow[t]{2}{*}{ Uso de medicamentos } & $\mathrm{Si}$ & 93 & 100 \\
\hline & No & 7 & 0 \\
\hline \multirow[t]{2}{*}{ Uso de oxígeno } & Sí & 57 & 77 \\
\hline & No & 43 & 23 \\
\hline
\end{tabular}

Tabla 2. Análisis descriptivo por edad según grupo de estudio

\begin{tabular}{|c|c|c|}
\hline Edad & Grupo experimental & Grupo control \\
\hline Mínimo & 54 & 61 \\
\hline Mediana & 71.0 & 75.0 \\
\hline Máximo & 90 & 89 \\
\hline Media & 73 & 75 \\
\hline SD & 10.1 & 7.5 \\
\hline
\end{tabular}

Análisis intergrupal

Con respecto a la medición pre-intervención, como se observa en la Tabla 3, existe un menor compromiso en las dimensiones de impacto y síntomas, así como una baja puntuación global del instrumento con un mínimo de 9.2 y un máximo de 74.6, para el grupo experimental, y un mínimo de 16.2 y un máximo de 76.3, para el grupo control, lo cual de acuerdo a la interpretación del cuestionario SGRQ, refleja una mejor calidad de vida, así como tener en cuenta que esta última es inversamente proporcional al puntaje del instrumento. 
En el caso de la dimensión de actividad, se observa una tendencia hacia puntuaciones altas en el grupo control y poco marcada, lo mismo sucede en el grupo experimental, lo que demuestra para los dos grupos, un mayor compromiso en la ejecución de las actividades de la vida diaria, por la disnea que produce la EPOC.

Tabla 3. Comparación de los grupos en la medición pre-intervención

\begin{tabular}{lccc}
\hline $\begin{array}{c}\text { Dimensiones } \mathbf{y} \\
\text { puntuación global }\end{array}$ & Grupo Experimental & Grupo Control & P \\
\hline Síntomas & $38+/-18$ & $42+/-17$ & 0.375 \\
\hline Actividad & $50+/-18$ & $64+/-18$ & 0.006 \\
\hline Impacto & $24+/-17$ & $31+/-23$ & 0.391 \\
\hline SGRQ global & $34+/-16$ & $43+/-19$ & 0.117 \\
\hline
\end{tabular}

Vale la pena resaltar, que no se encontró diferencia estadísticamente significativa entre ambos grupos en las dimensiones de síntomas e impacto, ni en la puntuación global del instrumento durante la primera medición, en términos generales se puede afirmar que la calidad de vida basal de ambos grupos es similar.

En la comparación de los grupos durante la medición pos-intervención, como se muestra en la Tabla 4, se observa alguna similitud en el comportamiento de la puntuación en las dimensiones de síntomas e impacto, las cuales se inclinan hacia valores bajos, lo que evidencia un menor compromiso en la salud derivado de la aparición de síntomas respiratorios y sus consecuencias sobre el funcionamiento social y psicológico.

En relación con la dimensión de actividad, las puntuaciones tienden a agruparse sobre valores bajos en el grupo experimental y valores altos en el grupo control, lo cual muestra menores limitaciones en el desarrollo de las actividades cotidianas en los pacientes que pertenecen al grupo experimental, comportamiento similar al presentado en la comparación entre grupos para la medición pre-intervención. De acuerdo a la puntuación global del instrumento, el grupo experimental reveló una mejor calidad de vida con un puntaje máximo de 75.2 y un mínimo de 6.1, en comparación con el grupo control cuyo puntaje máximo fue 79.1 y mínimo de 13.7.

Tabla 4. Comparación de los grupos en la medición pos-intervención

\begin{tabular}{lccc}
\hline $\begin{array}{c}\text { Dimensiones y } \\
\text { puntuación global }\end{array}$ & Grupo Experimental & Grupo Control & P \\
\hline Síntomas & $39+/-16$ & $45+/-16$ & 0.157 \\
\hline Actividad & $46+/-19$ & $56+/-16$ & 0.034 \\
\hline Impacto & $22+/-16$ & $30+/-22$ & 0.141 \\
\hline SGRQ global & $32+/-16$ & $41+/-18$ & 0.065 \\
\hline
\end{tabular}

Esto refleja que una excelente actividad educativa grupal de enfermería no es suficiente, para generar un impacto positivo sobre la calidad de vida de los pacientes con EPOC, aunque ésta aborde los lineamientos fundamentales del manejo y proporcione a los pacientes información escrita para reforzar las temáticas.

\section{Análisis intragrupal}

En relación a la comparación de las mediciones pre y pos-intervención al interior de cada uno de los grupos, no se encontraron cambios estadísticamente significativos en ninguno de los dos, como se evidencia en la Tabla 5, que ni la actividad educativa grupal de enfermería, ni la intervención individual usual de la institución de salud producen mejoría relevante en la calidad de vida de las personas con EPOC. 
Tabla 5. Análisis intra-grupal de los dos grupos. Comparación mediciones pre y pos-intervención

\begin{tabular}{lcc}
\hline & Grupo experimental & Grupo control \\
\hline Variable & Valor-p & Valor-p \\
\hline Síntomas & 0.807 & 0.538 \\
\hline Actividad & 0.285 & 0.125 \\
\hline Impacto & 0.222 & 0.719 \\
\hline Escala global & 0.199 & 0.586 \\
\hline
\end{tabular}

No obstante, es importante mencionar que los dos grupos mostraron una reducción de dos puntos en el promedio del puntaje total del instrumento, en la medición pos- intervención, así como una reducción marcada en los puntajes de la dimensión de actividad en esta misma medición, que si bien no implica un cambio estadísticamente significativo, si se traduce en la mejoría clínica de los pacientes que padecen este importante problema de salud pública.

\section{Discusión}

Las variables sociodemográficas como el sexo, la edad y la escolaridad, entre otras, son aspectos que los profesionales de enfermería deben considerar para el manejo del paciente con EPOC, ya que tienen la capacidad de influenciar la adherencia al tratamiento y con ello el control de la enfermedad, lo que finalmente repercute sobre la percepción de la calidad de vida ${ }^{25}$.

Los hallazgos del presente estudio se correlacionan con las estadísticas nacionales y mundiales sobre la EPOC, en donde se hace evidente la prevalencia en individuos mayores de 40 años $^{26}$. Los estudios a nivel mundial revelan que la EPOC se presenta en mayor medida en el género masculino ${ }^{5,27}$, sin embargo, en Colombia los resultados de esta investigación al igual de los reportados en el estudio Prepocol ${ }^{28}$, expresan un predominio del género femenino, que puede estar relacionado con la exposición a biomasa, por el contacto con el humo de leña y el incremento del tabaquismo en las mujeres. Con respecto a las demás variables, los resultados son coherentes con el estudio de Tülüce ${ }^{29}$ en el cual predomina ser casado, en los estudios de Posada et al. ${ }^{30}$ y Tiemensma et al. ${ }^{31}$ se destaca un máximo nivel educativo de bachillerato y, en la investigación de Cano-Rosales et al. ${ }^{32}$ se advirtió un predominio de estratos socioeconómicos bajos. De acuerdo con Rosińczuk et al. ${ }^{33}$, una mayor escolaridad puede influenciar el entendimiento de la condición de salud y los tratamientos, la aceptación de la enfermedad, así como el cumplimiento de las indicaciones proporcionadas por el personal de salud; de forma similar, Gerson y Mitravilles citados por Cano-Rosales ${ }^{32}$, encontraron en el estrato socioeconómico un factor de riesgo para el desarrollo de complicaciones relacionadas con la enfermedad, que puede asociarse con déficit nutricional, hacinamiento y exposición a contaminantes ambientales, que genera un efecto directo sobre la percepción de la calidad de vida.

Por su parte, la calidad de vida como un indicador del efecto de la enfermedad y su tratamiento sobre la función de los individuos ${ }^{8}$, plantea entonces la medición de la calidad de vida como una forma de evaluar los resultados en salud ${ }^{34}$ en este caso particular el efecto de la actividad educativa grupal.

En relación con la comparación de los grupos en la medición pre-intervención se encontró una diferencia estadísticamente significativa en la dimensión de actividad, que muestra para el grupo control un mayor compromiso en este aspecto. Este comportamiento se mantuvo al comparar los grupos en la medición pos-intervención, que implica una diferencia marcada en relación con las repercusiones que genera la enfermedad, sobre el desarrollo de las actividades de la vida diaria. Dichos hallazgos son similares a la investigación de Posada et al. ${ }^{30}$, señala que la dimensión más comprometida fue la de actividad con una media de 54\%; de acuerdo con Kyeong ${ }^{35}$ la inactividad física es común en los pacientes con EPOC, esta se presenta desde los estadios iniciales de la enfermedad, incluso cuando todavía no se han desencadenado los síntomas respiratorios, progresa en la medida en la que avanza la patología, lo que 
ocasiona un incremento del riesgo de exacerbaciones y hospitalizaciones, lo cual genera un impacto sobre la calidad de vida de los pacientes.

Con respecto al análisis intergrupal en la medición pos-intervención, los resultados del estudio no son congruentes con los reportes de la literatura, dado que investigaciones como la realizada por Bourne et al. ${ }^{36}$. encontraron una mejoría en la calidad de vida de los pacientes con EPOC, a partir de la implementación de un programa de autocuidado basado en grupos, en el que se desarrollaron cinco sesiones educativas para tratar temas relacionados con la definición de la enfermedad, el manejo de la respiración, el ahorro de la energía, el mantenimiento del ejercicio, así como el manejo de estrés y las emociones. De forma similar, Tülüce y Kutlutürkan ${ }^{29}$ encontraron una diferencia estadísticamente significativa en la calidad de vida tras la implementación de 12 sesiones de coaching en salud, y el estudio de Mozaffari et al. ${ }^{37}$ mostró que la implementación de un paquete educativo basado en la implementación de tres sesiones en grupos, tiene un efecto significativo sobre la calidad de vida de los pacientes con EPOC, en las dimensiones de función física, salud mental, estatus social, desempeño emocional, salud general, energía y vitalidad.

A pesar de que las temáticas abordadas en la sesión educativa de la presente investigación contemplaron las directrices planteadas en la literatura, la ausencia de mejoría estadísticamente significativa en la calidad de vida de los pacientes del grupo experimental, puede relacionarse con la cantidad de sesiones educativas desarrolladas, dado que la presente investigación sólo contó con una actividad educativa grupal, en estudios recientes se demuestra efectividad tras la implementación de tres o más sesiones, pues permite realizar un abordaje más profundo y un seguimiento más cercano a los pacientes. En este sentido, Cannon et al. ${ }^{38}$ encontró en su metaanálisis que las actividades educativas en los programas de autocuidado son las que mayor impacto producen sobre la calidad de vida del paciente con EPOC, ya que mejora, de forma significativa las dimensiones medidas con el cuestionario SGRQ, sin embargo, resalta también que la heterogeneidad y la falta de estandarización de las intervenciones crean ambivalencia acerca del contenido que se debe incluir, así como la duración más apropiada.

En relación al análisis intragrupal, ninguno de los grupos mostró cambios estadísticamente significativos en la comparación de las mediciones de calidad de vida pre y pos-intervención, aunque, en la medición pos-intervención del grupo experimental se encontró un menor incremento en la puntuación de la dimensión de síntomas y un descenso mayor en la puntuación de la dimensión de impacto, aún sin llegar a alcanzar la reducción de cuatro puntos que se consideran significativos en términos de mejoría en la calidad de vida para la escala global.

La dimensión de síntomas fue la única que demostró un incremento en la medición pos-intervención para los dos grupos, por consiguiente se evidencia un empeoramiento de la frecuencia y severidad de la sintomatología de la enfermedad, en este sentido, el estudio de Miravitlles ${ }^{5}$ reveló que más del 60\% de los pacientes con EPOC, experimentan uno o más síntomas en algún momento durante las 24 horas del día, lo cual se asocia a una peor percepción de los pacientes con respecto a sus resultados en salud.

Finalmente, vale la pena resaltar la reducción de dos puntos en el resultado global del Cuestionario SGRQ, tanto para el grupo control como para el experimental, que si bien, no existe un cambio estadísticamente significativo, existe una mejoría tanto clínica como en la percepción de la calidad de vida de los pacientes. Por esta razón, se hace necesario de parte de la disciplina de enfermería, la investigación constante sobre el cuidado de las personas con EPOC, con el objetivo de promover el desarrollo de habilidades de autocuidado ${ }^{20}$ las cuales deben desencadenar un mejor control de los síntomas y con ello una mejoría de las dimensiones de la calidad de vida. Acorde con el Instituto Nacional para la Salud y Cuidado de Excelencia, los síntomas y las manifestaciones de la EPOC pueden ser modificados con la adopción de comportamientos saludables, que incluyen ejercicio, cesación del hábito tabáquico, manejo de la ansiedad, control de la respiración, adherencia a la medicación y manejo de las exacerbaciones ${ }^{39}$, por lo que se hace imprescindible continuar investigando, además de implementar estrategias que favorezcan la calidad de vida de los pacientes con EPOC. 


\section{Conclusiones}

La aplicación de una sesión educativa grupal de enfermería para pacientes con EPOC, no generó cambios estadísticamente significativos en la calidad de vida de los mismos, a pesar de que demostró una disminución de dos puntos en el resultado global del instrumento.

En la medición pos-intervención del grupo experimental, se observó una mejoría en las dimensiones de actividad e impacto y un deterioro menos marcado en la dimensión de síntomas con respecto al grupo control, lo que demuestra una mejoría clínica de los pacientes.

Se requiere mayor investigación de enfermería en el área, con el objetivo de perfeccionar las intervenciones de cuidado tendientes a mejorar la calidad de vida de los pacientes con EPOC, con ello reducir el impacto de este importante problema de salud pública.

Sería deseable hacer estudios con población cautiva, o la inclusión de tecnologías de la información y la comunicación, para los pacientes que por diversas circunstancias no pueden desplazarse a los centros asistenciales. Asimismo, debe evaluarse la realización de una mayor cantidad de actividades educativas

\section{Limitaciones}

La dificultad para la conformación estándar de los grupos requeridos para el desarrollo de la actividad educativa grupal, se considera como el principal factor que pudo haber condicionado el resultado de la investigación, dado que la edad de los pacientes, el uso de oxígeno permanente y la ausencia de una adecuada red de apoyo, impidió el desplazamiento de los pacientes y la asistencia a la actividad programada. Adicionalmente, la mayoría de los pacientes con diagnóstico de EPOC, por historia clínica, no cuentan con pruebas de función pulmonar, lo que limita el análisis de los datos en relación con la severidad de la enfermedad.

\section{Responsabilidades éticas}

Protección de personas y animales. Los autores declaran que en este estudio no se realizaron experimentaciones en seres humanos ni animales.

Confidencialidad de datos. Los autores declaran que en el artículo no aparecen datos de pacientes.

Derecho a la privacidad y consentimiento informado. Los autores han obtenido el consentimiento informado de los sujetos referidos en el artículo. Este documento está en poder del autor de correspondencia. Conflicto de intereses. Los autores declaran no tener conflicto de intereses.

Financiamiento. Pontificia Universidad Javeriana-Bogotá.

Agradecimientos. A la Fundación Hospital San Carlos de la ciudad de Bogotá-Colombia, por su participación en la investigación.

\section{Referencias}

1. Global initiative for chronic obstructive lung disease. Global strategy for the diagnosis, management, and prevention of chronic obstructive pulmonary disease. (2018 report). Wisconsin: GOLD; 2018 Nov 20 [Consultado octubre 21 2018]. p. 142. Disponible en: http://bit.ly/REUgold

2. Institute for Health Metrics and Evaluation. Global Burden of Disease (GBD). Washington, USA: University of Washington; 2017 [Consultado 2018 octubre 21]. Disponible en: http://bit.ly/REUihme

3. Naghavi M, Abajobir AA, Abbafati C, Abbas KM, Abd-Allah F, Abera SF, et al. Global, regional, and national age-sex specific mortality for 264 causes of death, 1980-2016: a systematic analysis for the Global Burden of Disease Study 2016. Lancet. 2017; 390(10100): 1151-210. https://doi.org/10.1016/S0140-6736(17)32152-9

4. Merino M, Villoro R, Hidalgo-Vega A, Carmona C, Grupo deTrabajo Colaborativo EPOC-Extremadura. Estimación de la carga económica y social de la EPOC en Extremadura. Madrid: Fundación Weber; 2017. 
5. Miravitlles M, Worth H, Soler-Cataluña JJ, Price D, De Benedetto F, Roche N, et al. Observational study to characterize 24-hour COPD symptoms and their relationship with patient-reported outcomes: results from the ASSESS study. Respir Res. 2014; 15 (1): [13 p.].

https://doi.org/10.1186/s12931-014-0122-1

6. Costa X, Gómez-Batiste X, Pla M, Martinez-Muñoz M, Blay C, Vila L.Vivir con la enfermedad pulmonar obstructiva crónica avanzada: el impacto de la disnea en los pacientes y cuidadores. Aten. prim. 2016; 48 (10): 665-73. http://dx.doi.org/10.1016/j.aprim.2016.02.008

7. Okutan O, Tas D, Demirer E, Kartaloglu Z. Evaluation of quality of life with the chronic obstructive pulmonary disease assessment test in chronic obstructive pulmonary disease and the effect of dyspnea on disease-specific quality of life in these patients. Yonsei Med J. 2013; 54(5): 1214-9. http://dx.doi.org/10.3349/ymj.2013.54.5.1214

8. Saeid-Pour J, Jafari M, Ghazi-Asgar M, Dayani-Dardashti H, Teymoorzadeh E. The impact of self-care education on life quality of diabetic patients. JHA. 2013; 16(52): 26-36.

http://jha.iums.ac.ir/article-1-1227-en.pdf

9. Muñoz-Cobos F, Acero-Guasch N, Cuenca-del-Moral R, Barnestein-Fonseca P, Leiva-Fernández F, García-Ruiz A. Cómo vivir con EPOC: percepción de los pacientes. An. psicol. 2016; 32(1): 18-31. http://dx.doi.org/10.6018/analesps.32.1.186211

10. Durán-Montes LA, Cisneros-Sandoval FJ, Gutiérrez-Román EA. Calidad de vida en enfermedad pulmonar obstructiva crónica: experiencia de un hospital del occidente, México. Rev. Med. Inst. Mex. Seguro Soc. 2015; 53(3): 380-5.

11. Mata-Hernández MC. La educación terapéutica para mejorar la adhesión en la enfermedad pulmonar obstructiva crónica (EPOC). Monogr. arch. bronconeumol. 2016; 3(8): 213-17.

12. Soler JJ, Martínez-García MA, Román P, Orero R, Terrazas S, Martínez-Pechuán A. Eficacia de un programa específico para pacientes con EPOC que presentan frecuentes agudizaciones. Arch. bronconeumol. 2006; 42(10): 501-8. https://doi.org/10.1157/13093392

13. Garrote-Garrote A, Del Cojo-Arroyo T. Educación grupal para la salud, reto o realidad. España: Ediciones Díaz de Santos. 2015. p.8.

14. Alves-Pereira D, Da Silva-Campos-Costa NM, Lima-Sousa AL, Brandão Veiga-Jardim PC, De OliveiraZanini CR. Efectos de intervención educativa sobre el conocimiento de la enfermedad en pacientes con diabetes mellitus. Rev. Lat.-Am. Enferm. 2012; 20(3): p.8.

http://dx.doi.org/10.1590/S0104-11692012000300008

15. Fernández AR, Manrique-Abril FG. Efecto de la intervención educativa en la agencia de autocuidado del adulto mayor hipertenso de Boyacá, Colombia, Suramérica. Cienc. enferm. 2010; 16(2): 83-97. http://dx.doi.org/10.4067/S0717-95532010000200009

16. Rodríguez-Martín C, Castaño-Sánchez C, García-Ortiz L, Recio-Rodríguez JI, Castaño-Sánchez Y, Gómez-Marcos MA. Eficacia de una intervención educativa grupal sobre cambios en los estilos de vida en hipertensos en atención primaria: un ensayo clínico aleatorio. Rev. esp. salud pública 2009; 83(3): 441-52.

17. Aresté-Albà N, Torres-Puig Gros J. Eficacia de una intervención educativa a pacientes con enfermedad pulmonar obstructiva crónica y sus cuidadores. Metas enferm. 2017; 20(1): 50-6.

18. Sánchez-Alonso RI. Efectividad de una intervención educativa grupal en atención primaria para mejora de la calidad de vida de los pacientes con EPOC sobre el correcto manejo de los dispositivos inhaladores. [Tesis]. España: Facultad de Medicina-Universidad Miguel Hernández de Eche; 2017.

19. Folch A, Orts-Cortés MI, Hernández-Carcereny C, Seijas-Babot N, Maciá-Soler L. Programas educativos en pacientes con enfermedad pulmonar obstructiva crónica. Revisión integradora. Enfermería glob. 2017; 16(1): 537-55. https://doi.org/10.6018/eglobal.16.1.249021 
20. Zwerink M, Brusse-Keizer M, van derValk PDLPM, Zielhuis GA, Monninkhof EM, van der Palen J, Frith PA, et al. Self management for patients with chronic obstructive pulmonary disease (Review). Cochrane Database of Systematic Reviews. Cochrane Library. 2014; (3): p.159. https://doi.org/10.1002/14651858.CD002990.pub3

21. Grupo de trabajo de GesEPOC. Guía de Práctica Clínica para el Diagnóstico y Tratamiento de Pacientes con Enfermedad Pulmonar Obstructiva Crónica (EPOC)-Guía Española de la EPOC (GesEPOC), Versión 2017. Arch Bronconeumol. 2017; 53(Supl 1): 2-64. https://doi.org/10.1016/S0300-2896(17)30356-3

22. Schünemann HJ, Griffith L, Jaeschke R, Goldstein R, Stubbing D, Guyatt GH. Evaluation of the minimal important difference for the feeling thermometer and the St. George's Respiratory Questionnaire in patient with chronic airflow obstruction. J Clin Epidemiol. 2003; 56(12): 1170-6.

https://doi.org/10.1016/S0895-4356(03)00115-X

23. Sieloff CL, Messmer PR. Imogene M. King: Marco de sistemas de interacción y teoría intermedia de la consecusión de objetivos. En: Alligood MR, Marriner A. Modelos y teorías en Enfermería. España: Ediciones Elsevier. 8 $8^{\mathrm{va}}$ ed. 2015.

24. Ferrer M, Alonso J, Prieto L, Plaza V, Monsó E, Marrades R, et al. Validity and reliability of the St George's Respiratory Questionnaire after adaptation to a different language and culture: The Spanish example. Eur Respir J. 1996; 9(6): 1160-6. https://doi.org/10.1183/09031936.96.09061160

25. Pineda-Higuita SE, Ramos-Melchor VJ, Cadavid-Carmona D. Calidad de vida en pacientes con Enfermedad Pulmonar Obstructiva Univ. Salud. 2016; 18(3): 482-93. http://dx.doi.org/10.22267/rus.161803.53

26. Farag TS, Sobn ESM, Elsawy SB, Fahmy BM. Evaluation of health-related quality of life in patinets with chronic obstructive pulmonary disease. Egypt J Bronchol. 2018; 12(3): 288-94. https://doi.org/10.4103/ejb.ejb_11_18

27. Folch-Ayora A, Orts-Cortés MI, Macia-Soler L, Andreu-Guillamon MV, Moncho J. Patient education during hospital admission due to exacerbation of chronic obstructive pulmonary disease: Effects on quality of life - Controlled and randomized experimental study. Patient Educ Couns. 2018; 102(3): 511-9. https://doi.org/10.1016/j.pec.2018.09.013

28. Caballero A, Torres-Duque CA, Jaramillo C, Bolivar F, Sanabria F, Osorio P, et al. Prevalence of COPD in five colombian cities situated at low, medium and high altitude. (PREPOCOL study). Chest. 2008; 133(2): 343-9. https://doi.org/10.1378/chest.07-1361

29. Tülüce D, Kutlutürkan S. The effect of health coaching on treatment adherence, self-efficacy, and quality of life in patients with chronic obstructive pulmonary disease. Int J Nurs Pract. 2018; 24(4): p.11. https://doi.org/10.1111/ijn.12661

30. Posada A, Caballero A, Ibáñez M, Ardila L, Álvarez A, Soler S, et al. Evaluación de la calidad de vida en pacientes del programa manejo integral de la enfermedad pulmonar obstructiva crónica (EPOC) de la EPS sanitas en Bogotá. Rev. Médica Sanitas. 2009; 12(2): 14-21.

31. Tiemensma J, Gaab E, Voorhaar M, Asijee G, Kaptein AA. Illness perceptions and coping determine quality of life in COPD patients. Int J Chron Obstruct Pulmon Dis. 2016; 11(1): 2001-7. https://doi.org/10.2147/COPD.S109227

32. Cano-Rosales DJ, Bolívar-Grimaldos F, Omaña-Roa JJ, Sepúlveda-Sierra AM. Calidad de vida relacionada con la salud en pacientes con enfermedad pulmonar obstructiva crónica en Hospital Universitario de Santander. MÉD.UIS. 2017; 30 (2):11-9. http://dx.doi.org/10.18273/revmed.v30n2-2017001

33. Rosińczuk J, Przyszlak M, Uchmanowicz I. Sociodemographic and clinical factors affecting the quality of life of patients with chronic obstructive pulmonary disease. Int J Chron Obstruct Pulmon Dis. 2018; 13: 2869-82. https://doi.org/10.2147/COPD.S165714 
34. Fernández-López JA, Fernández-Fidalgo M, Cieza A. Los conceptos de calidad de vida, salud y bienestar analizados desde la perspectiva de la clasificación internacional del funcionamiento (CIF). Rev. esp. salud pública. 2010; 84(2): 169-84.

35. Kyeong-Cheol S. Physical activity in chronic obstructive pulmonary disease: clinical impact and risk factors. Korean J Intern Med. 2018; 33(1): 75-7. https://doi.org/10.3904/kjim.2017.387

36. Bourne CLA, Kanabar P, Mitchell K, Schreder S, Houchen-Wolloff L, Bankart MJG, et al. A selfmanagement programme of activity coping and education-SPACE for COPD $(\mathrm{C})$ - in primary care: The protocol for a pragmatic trial. BMJ Open. 2017; 7(7): p.10. http://dx.doi.org/10.1136/bmjopen-2016-014463

37. Mozaffari M, Azami S, Naderi M. Determining the effect of implementing an educational package on quality of life among patients with chronic obstructive pulmonary disease referring to teaching hospitals affiliated with Ilam University of Medical Sciences in 2016. J Family Med Prim Care. 2018; 7(3): 606-11. https://doi.org/10.4103/jfmpc.jfmpc_304_17

38. Cannon D, Buys N, Bajee-Sriram K, Sharma S, Morris N, Sun J. The effects of chronic obstructive pulmonary disease self-management interventions on improvement of quality of life in COPD patients: A meta-analysis. Respir Med. 2016; 121: 81-90. http://dx.doi.org/10.1016/j.rmed.2016.11.005

39. National Institute for Health and Care Excellence. Chronic obstructive pulmonary disease in over 16s: diagnosis and management (CG101). Manchester, UK: NICE; 2010 June. [Consultado octubre 20 2018]. Disponible en: http://bit.ly/reuCG101 